\title{
Morsellized Allograft and Acetabular Reconstruction Cage: Follow - up of 2 to 9 years
}

\author{
AA Abbas, MS (Ortho)*, AM Merican, MS (Ortho)*, JA Mohamad, MS (Ortho)** \\ *Department of Orthopaedic Surgery, Faculty of Medicine, University of Malaya, Kuala Lumpur, Malaysia \\ **Selangor Medical Centre, Shah Alam, Selangor, Malaysia
}

\begin{abstract}
We report the outcome of 32 patients who underwent total hip replacement (THR) augmented with morsellized fresh frozen femoral head allografts and acetabular reconstruction cages. Nine patients underwent primary THR and 23 patients underwent revision THR. Follow-up ranged from two to 9 years. Two most common indications for the procedures as reported in literature were rheumatoid arthritis and aseptic loosening of the hip. All but one patient achieved good outcome with radiographs showing full incorporation of bone grafts and no evidence of loosening of the implants. Morsellized bone grafting used with acetabular reinforcement devices is valuable for addressing severe acetabular deficiencies.
\end{abstract}

Key Words:

Total hip replacement, Acetabular bone deficiency, Acetabular reconstruction cage

\section{INTRODUCTION}

Total hip replacement (THR) in a patient with acetabular bone deficiency remains a major challenge for orthopaedic surgeons. Such condition may present in primary or revision THR setting and the degree of bone loss can be massive.

There has been a growing trend supporting cementless fixation using oversized cups for acetabular revision. Although this is a viable option for patients with sufficient bone stock, it is difficult to obtain stable fixation when bone stock is deficient. One study reported a $24 \%$ rate of loosening with the use of a bi-lobed acetabular cup in patients with extensive bone deficiency ${ }^{1}$. Another option is by using acetabular reinforcement with reconstruction cage and morsellized bone graft. Numerous studies have reported promising results with this technique in the medium and long term $^{2,3}$.

The aim of this study is to report the use of morsellized allograft and acetabular reconstruction cage in patients requiring total hip replacement in both the primary and revision settings. We would like to present the medium range outcomes of these patients.

\section{MATERIALS AND METHODS}

Between 1997 and 2004, there were a total of 32 patients (34 hips) who underwent total hip replacement with acetabular reconstruction utilizing morsellized allograft and cementless acetabular reconstruction cages. Nine patients (nine hips) underwent primary THR whereas the remaining 23 patients ( 25 hips) underwent revision THR. The patients ranged in age from 29 to 80 years, with an average of 64.3 years. All primary THR patients in this study were female. In the revision group, there were seven males and sixteen females.

The reasons for primary THR for patients in this study were acetabular protrusion of unknown cause in two patients, rheumatoid arthritis in four patients, avascular necrosis of femoral head in two patients and trauma in one patient. The reasons for revision THR were aseptic loosening in 22 patients and infection in one patient. Based on the AAOS Classification $^{4}$ (Fig. 1), eight hips had Type II defects, 17 had Type III defects and nine had Type IV defects.

Reconstruction cages used were either Bürch-Schneider, Müller or Ganz cages. Fresh frozen femoral heads (morsellized prior to insertion) were used to fill acetabular defects.

\section{RESULTS}

All patients returned for follow-ups. The average Harris Hip Score preoperatively was 30 , with a range of 22 to 43 . The majority of the patients reported marked preoperative pain. All patients were housebound preoperatively, and two were not able to walk due to pain.

Postoperatively, 31 patients (33 hips) reported good functional outcomes at the last follow-up (Range 2 to 9 years). Twenty-five patients were able to walk for at least 30 
minutes. Five patients still require a walking aid. All patients reported that their pain was much less compared to the preoperative levels. The average Harris Hip Score postoperatively was 76 (range of $61-89$ ). Radiographically, all hips showed full incorporation of bone graft. Acetabular components and cages showed no signs of loosening at last follow-up (Figures 2 and 3).
One failure occurred in a patient who had a previously irradiated pelvis for carcinoma of the cervix. She fell two years after the hip replacement and sustained fracture of the pelvis. The patient underwent revision surgery, but postoperative recovery was complicated by infection and subsequently the implant loosened. Eventually Girdlestone procedure was performed for the affected hip.

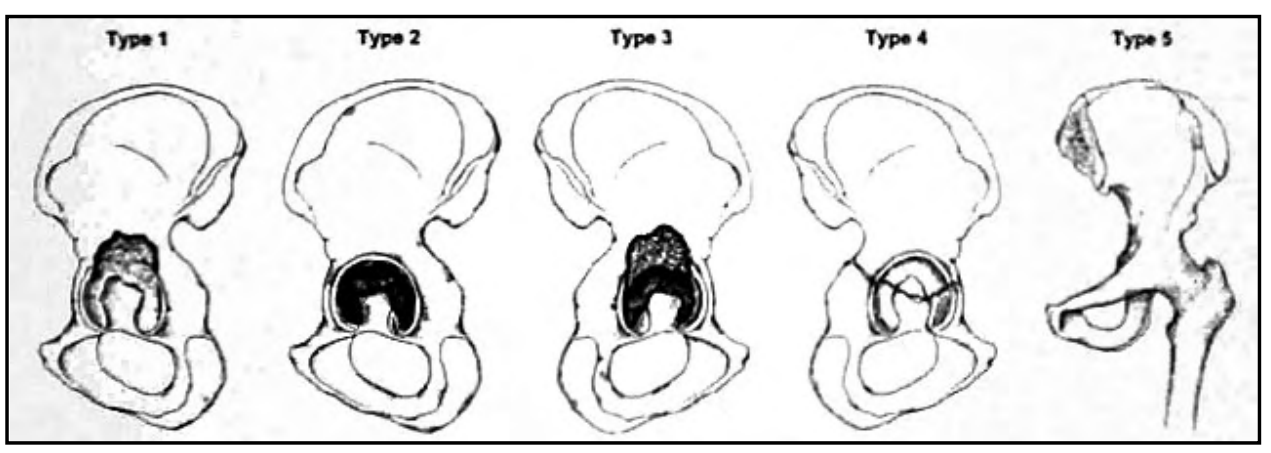

Fig. 1: Classification of acetabular defects according to the American Academy of Orthopaedic Surgeons Committee on the Hip. ${ }^{4}$ The five types of defects are segmental (Type 1), cavitary (Type 2), combined segmental and cavitary (Type 3), pelvic dissociation (Type 4) and hip fusion (Type 5).

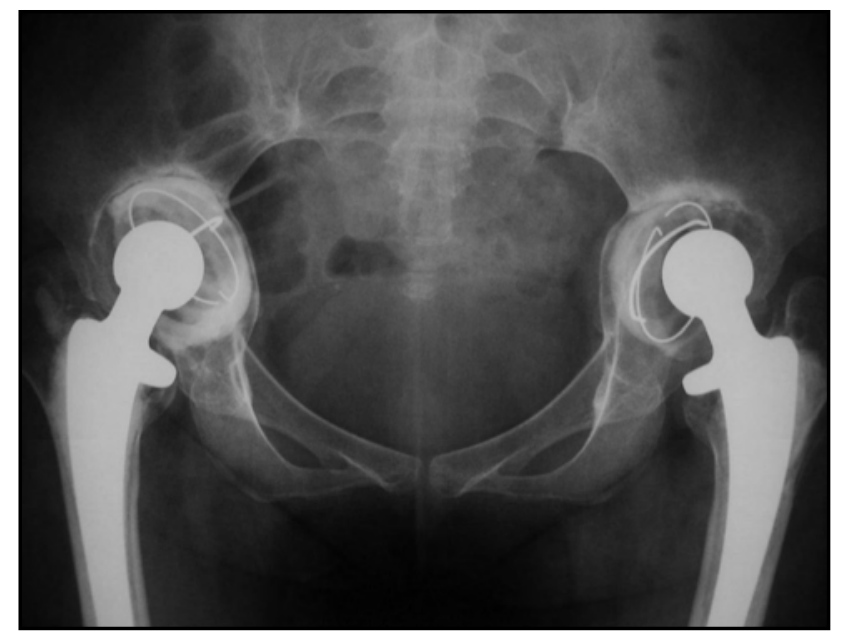

Fig. 2A: Preoperative radiograph of bilateral hip arthroplasty with loosening.

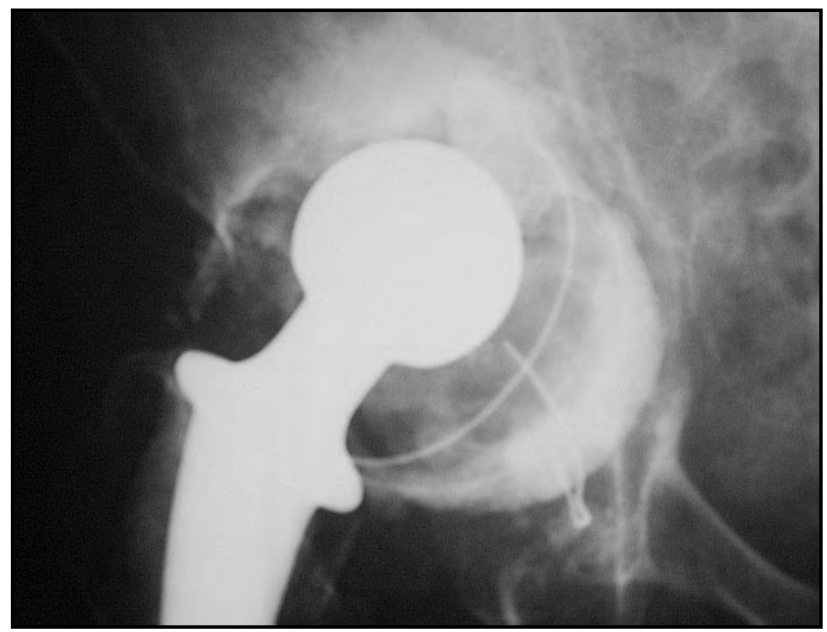

Fig. 3a: Preoperative radiograph of a hip requiring revision arthroplasty.

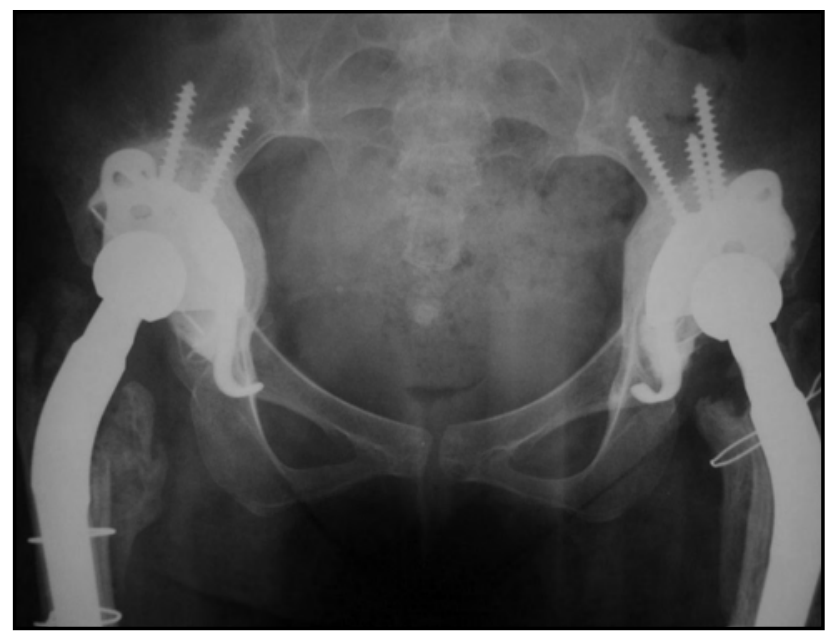

Fig. 2b: Plain radiograph of both hips 7 years after surgery.

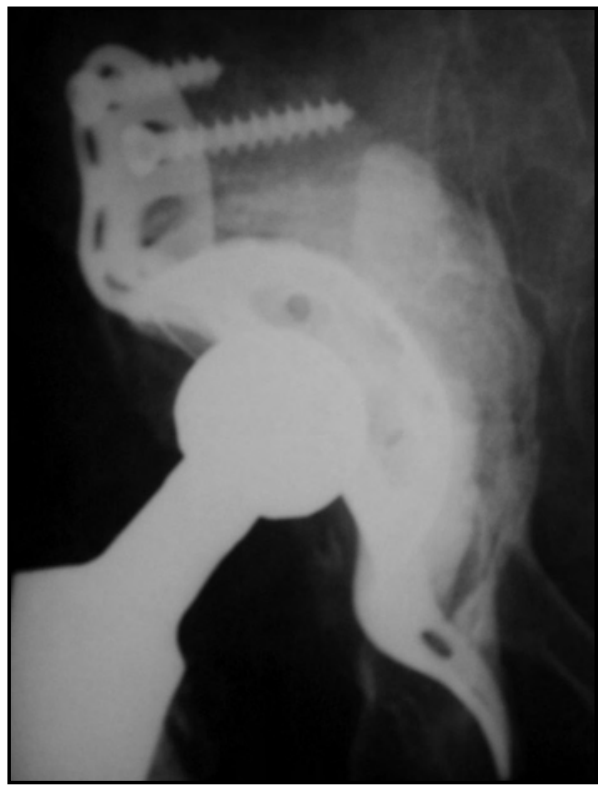

Fig. 3b: Plain radiograph of the hip 5 years after revision surgery. 


\section{DISCUSSION}

Acetabular reconstruction with reinforcement cage has been advocated for total hip replacement in patients with significant bone loss. Deficiency of the acetabular bone makes implantation of new acetabular cup difficult. Normal placement of the implants help to normalize the hip centre and acetabular biomechanics, ${ }^{2,5}$. They also protect the bone grafts during integration and are instrumental in aiding restoration of bone stock ${ }^{5,6}$. A cage is indicated when there is less than $50 \%$ of viable, stable host bone to support and stabilize the components?

Contained defects where the acetabular columns and rim are intact account for most acetabular revisions. Allograft may be required to fill the bone defects and restore the shape of the acetabulum. Once fully incorporated, bone stock will be restored $^{8}$. The type of allograft used depends on the degree of bone loss. Morsellized cancellous bone graft is used in contained acetabular defects9. In this study, radiographs of all the patients at the last follow-up showed full incorporation of the bone grafts. Radiographs of the patient who was considered a failure due to subsequent fracture after a fall had radiological evidence of bone graft incorporation before the incident.

Result of surgery using reconstruction cages is welldocumented ${ }^{3,5,10,11}$. However, most available studies report on the use of acetabular reconstruction cages in the revision THR. In our study, acetabular reconstruction cages and morsellized bone grafts were also used in nine primary THR (26\%) for those with significant acetabular bone defects. Functional outcome in the primary THR were comparable to that of the revision group.

\section{REFERENCES}

1. Chen WM, Jr CAE, Hopper RH: Acetabular revision with use of a bilobed component inserted without cement in patients who have acetabular bone stock deficiency. J Bone Joint Surg 2000; 92A: 197-206.

2. Gill TJ, Sledge JB, Muller ME: The Burch-Schneider anti-protrusio cage in revision total hip arthroplasty: Indications, Principles and Long-Term Results. J Bone Joint Surg 1998; 80B: 946.

3. Schlegel UJ, Aldinger PR, H Mau, Breussch SJ: Acetabular Reinforcement Rings in Revision Hip Arthroplasty: 1-15 Year Followup of Muller-, Ganz- and Burch-Schneider Rings. J Bone Joint Surg 2004; 86B: 275.

4. D'Antonio JA, Capello WN, Borden LS: Classification and management of acteabular abnormalities in total hip arthroplasty. Clin Orthop 1989; 243: 126-37.

5. Schatzker J, Wong MK: Acetabular Revision: The Role of Rings and Cages. Clin Orthop 1999; 369: 187-97.

6. Gross AE, Goodman S: The Current Role of Structural Grafts and Cages in Revision Arthroplasty of the Hip. Clin Orthop 2004; 429: 193-200.

7. Cuckler JM, Moore KD: The Role of Acetabular Cages in Revision. Orthoped 2004; 27(8): 831.

8. vanderDonk S, Buma P, Slooff TJJH, Gardeniers JWM, Schreures BW: Incoporation of Morselized Bone Grafts: A Study of 24 Acetabular Biopsy Specimens. Clin Orthop 2002; 396: 131-41.

9. Paprosky WG, O'Rourke M, Sporer SM: The Treatment of Acetabular Bone Defects with an Associated Pelvic Discontinuity. Clin Orthop 2005; 441: 216-20.

10. Rosson J, Schatzker J: The use of reinforcement rings to reconstruct deficient acetabula. J Bone Joint Surg 1992; 74B: 716-20.

11. Winter E, Piert M, Volkmann R, et al. Allogeneic Cancellous Bone Graft and a Burch-Schneider Ring for Acetabular Reconstruction in Revision Hip Arthroplasty. 2001; 83(6): 862. 